\title{
Observation of anomalous Hall effect in thin film EuS
}

\author{
I. J. Guilaran, D. E. Read, R. L. Kallaher, P. Xiong, and S. von Molnár \\ Department of Physics and MARTECH, Florida State University, Tallahassee, Florida 32306, USA \\ P. A. Stampe and R. J. Kennedy \\ Department of Physics, Florida A\&M University, Tallahassee, Florida 32307, USA \\ J. Keller \\ Physikalisches Institut, RWTH Aachen, 52056 Aachen, Germany \\ (Received 24 April 2003; revised manuscript received 15 August 2003; published 17 October 2003)
}

\begin{abstract}
We report on a study of the magnetotransport properties of EuS thin films grown by electron-beam deposition on (100) GaAs and (100) Si. The films are naturally doped due to a varying degree of sulfur deficiency. The sulfur deficiency and thus the doping level is found to vary systematically with the growth temperature. In these disordered self-doped materials we observe a large nonlinear component in the Hall effect at low temperatures. The close scaling between the Hall data and the magnetization implies that this effect is an anomalous Hall effect rather than a change of carrier concentration with magnetic field. The extracted anomalous Hall coefficient is found to scale linearly with the resistivity, indicating that it is due to skew scattering of the conduction electrons by the defects.
\end{abstract}

DOI: 10.1103/PhysRevB.68.144424

PACS number(s): 75.50.Pp, 72.20.My, 72.20.Ht, 75.47.Pq

\section{INTRODUCTION}

There has been growing interest in extending the successful metal-based spintronics research into semiconductor systems in the hope of producing true three-terminal spintronic devices such as spin transistors. ${ }^{1,2}$ A major challenge in this field is electrical spin injection from a ferromagnet into a nonmagnetic semiconductor where the spins can be manipulated coherently and eventually detected. The best material choices for the ferromagnetic components in such devices are most likely magnetic semiconductors because of their compatibility in crystal and band structures, carrier density, and conductivity with conventional semiconductors. These qualities are shown to be important for efficient spin injection from a ferromagnet into a semiconductor.,

The europium chalcogenides have been studied extensively in bulk single crystal form, revealing a variety of spectacular magnetotransport properties such as giant negative magnetoresistance $e^{5,6}$ and the formation of bound magnetic polarons. ${ }^{7,8}$ Although EuS has a low $T_{c}(16.5 \mathrm{~K})$ making it unsuitable for practical devices at room temperatures, it is an excellent model system for proof-of-concept studies of spin injection into semiconductors. Doped EuS is potentially a $100 \%$ spin polarized spin injector, while thin insulating layers of EuS have been shown to be effective spin filters. ${ }^{9-12}$

Growth of high quality thin films and a good understanding of their structural and physical properties are a prerequisite for device applications. Previous work has shown that the composition, magnetic, optical, and transport properties of EuS films can be varied by growing the films on different substrates, ${ }^{13,14}$ at different growth temperatures, ${ }^{13-15}$ and by using different annealing procedures. ${ }^{16,17}$ In particular, it has been found that the stoichiometry of the EuS films can be controlled with the substrate temperature during growth, ${ }^{14,15,18}$ providing a convenient way of tuning the film conductivity without introducing extrinsic doping. A detailed investigation of the Hall effect in these films, however, has, to our knowledge, not previously been performed. In this paper we investigate the magnetic and magnetotransport properties, including the Hall effect of Eu-rich EuS thin films as a function of growth conditions and compare their characteristics with earlier work ${ }^{6}$ on bulk single crystals of EuS. We observe a clear correlation between the carrier density in the films and their growth temperatures, and we have found an anomalous Hall effect in these thin films that was not seen in the bulk material.

The Hall resistivity $\rho_{H}$ in ferromagnetic materials obeys the relation

$$
\rho_{H}=R_{0} B+\mu_{0} R_{S} M,
$$

where $R_{0}$ is the ordinary Hall coefficient, $B$ is the magnetic induction, $R_{S}$ is the anomalous Hall coefficient, $M$ is the magnetization, and $\mu_{0}$ is the permeability of free space. The $R_{0} B$ term arises from the ordinary Hall effect and, using a simple one-band model, can be related to the carrier concentration, $n$, through $R_{0}=(1 / n e)$. The second term, $\mu_{0} R_{S} M$, is the anomalous contribution, which arises from the spin-orbit interaction between the conduction electrons and scattering centers such as impurities and phonons. It is generally understood $^{19}$ that there are two possible scattering mechanisms responsible for the anomalous term: skew scattering, an asymmetrical deflection of the electrons from their original path, and side-jump scattering, a quantum mechanical transverse displacement of the electron trajectory. The quantitative difference between the two manifests itself in the relation

$$
R_{S} \propto \rho^{n},
$$

where $\rho$ is the sample resistivity, and $n=1$ for skew scattering while $n=2$ for side-jump scattering. 
Surprisingly, previous Hall effect measurements ${ }^{6}$ on single crystals of EuS have consistently shown a negligibly small anomalous component. Although nonlinearity was observed in the applied field $\left(H_{A}\right)$ dependence of the Hall voltage $\left(V_{H}\right)$, the nonlinearity was not interpreted as an anomalous Hall effect (AHE) but as a change in carrier concentration with increasing field. Since thin films have a demagnetization factor of $\cong 1$, they are an ideal system for distinguishing the ordinary and anomalous components in the Hall effect from measurements because the magnetic induction $B$ is equal to the applied field.

\section{EXPERIMENT}

EuS powder was synthesized from an $\mathrm{Eu}_{2} \mathrm{O}_{3}$ precursor via reaction with $\mathrm{H}_{2} \mathrm{~S}$ at $1100{ }^{\circ} \mathrm{C}$ in a tube furnace. The samples were reground into a powder and reacted repeatedly in order to ensure uniform formation of EuS. The powder targets were then used as the source material for an electronbeam heated tungsten crucible in an ultrahigh vacuum chamber with a base pressure of $10^{-9}$ Torr and a pressure of $10^{-8}$ Torr during evaporation. The samples on which magnetotransport measurements were performed were grown on either (100) GaAs, (100) Si, or (111) Si. Evaporation rates for the samples on GaAs were $2 \AA / s$ grown to a thickness of $470 \AA$. All of the samples were grown at substrate temperatures from -38 to $300^{\circ} \mathrm{C}$.

For the samples on GaAs on which we report transport measurements, two separate films were grown simultaneously in order to correlate the magnetic, structural, and transport measurements. These two films consisted of a Hall bar sample patterned by shadow masking for transport studies and an unpatterned sample for magnetic and x-ray characterization. The magnetic measurements were performed with a Quantum Design superconducting quantum interference device magnetometer which has a sensitivity of $10^{-7} \mathrm{emu}$, and both the $\theta-2 \theta$ and the pole figure $\mathrm{x}$-ray scans were performed on a PW3040 Phillips Materials Research Diffractometer. In order to accurately account for the large diamagnetic contribution in the magnetic measurements from the GaAs substrate, a similarly sized GaAs sample from the same wafer was measured to determine its susceptibility at relevant temperatures. The appropriate contribution was subtracted from the magnetic measurements of the EuS samples.

The magnetotransport measurements were performed using both dc and low frequency ac lock-in techniques and the results were found to be identical. $I-V$ curves were taken preceding all measurements in order to ensure that the contacts were Ohmic and that there was no Joule heating. No discrepancies in the data were found by repeating measurements using different magnetic field sweep rates. Except for the hysteretic effects in the magnetoresistance (MR) that will be discussed shortly, no dependence on the field sweep direction was observed. The Hall data were extracted by taking the asymmetric part of the voltage perpendicular to the current, $V_{y}$, as a function of $B$. The low-field hysteresis mentioned below in the MR was accounted for in the Hall data by averaging the data from both sweep directions
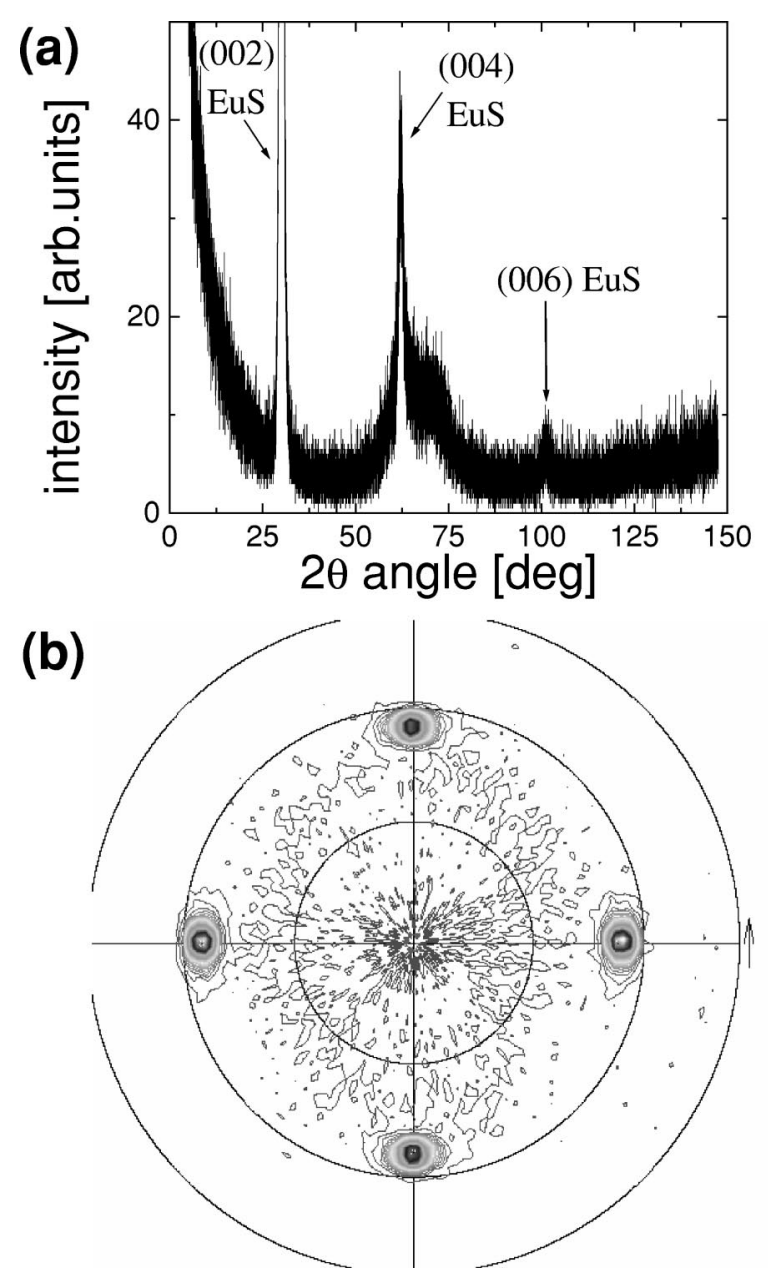

FIG. 1. (a) X-ray $\theta-2 \theta$ scan of a sample grown at $235 \mathrm{~K}$. The substrate peak has been removed except for the remnant at $70^{\circ}$. (b) Pole figure showing in-plane diffraction peaks for a sample grown on a surface treated (100) Si substrate.

$(+7.35 \mathrm{~T} \rightarrow-7.35 \mathrm{~T} \rightarrow+7.35 \mathrm{~T})$ before taking the asymmetric part; also, the hysteretic effect occurs at a field much lower than the saturation field of the samples and thus does not complicate our analysis of the Hall data.

\section{RESULTS AND DISCUSSION}

Stochiometric EuS has a simple cubic structure with a lattice constant of $5.96 \AA$ and a magnetic ordering temperature of $16.5 \mathrm{~K}$. X-ray $\theta-2 \theta$ scans show that films grown on (100) GaAs are highly oriented in the (100) direction, independent of the growth temperature. Figure 1(a) shows an example of such a diffraction pattern for a sample grown at room temperature. The in-plane order of the films was found to depend sensitively on the substrate preparation as revealed by pole figure measurements [Fig. 1(b)]: films grown on untreated substrates with a native oxide layer showed no inplane ordering, while removing the oxide layer on (100) $\mathrm{Si}$ substrates by a buffered HF etch immediately prior to growth resulted in pole figures with well defined spots indicating a high degree of in-plane orientation. 


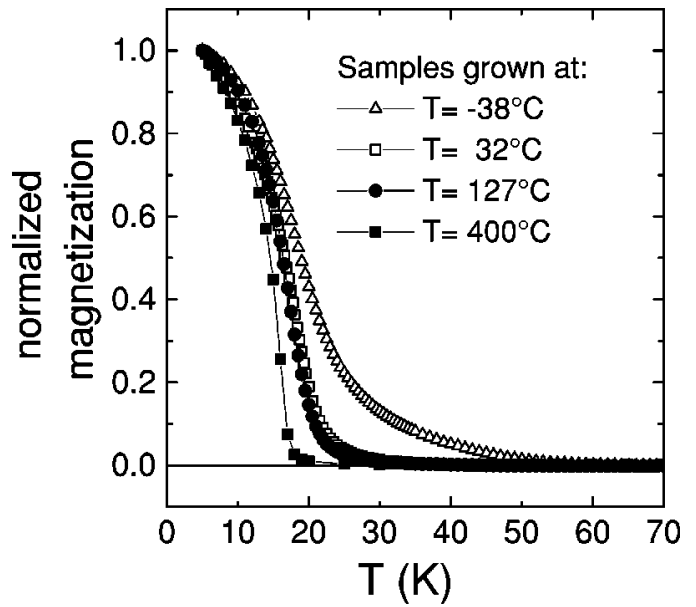

FIG. 2. Magnetization vs temperature curves for samples grown on (100) GaAs and (111) Si. The latter film exhibits a $T_{c}$ very near the bulk value of $16.5 \mathrm{~K}$.

Magnetic measurements were made with the applied magnetic field perpendicular to the film plane. A broadening of the ferromagnetic transition and an enhancement of the transition temperature $T_{c}$ with decreasing growth temperature is observed, as shown in Fig. 2. As the growth temperature is increased, $T_{c}$ becomes better defined and approaches the bulk ordering temperature. Previous studies, ${ }^{13,15}$ including Rutherford backscattering experiments, show that stochiometric films are formed at a substrate temperature of approximately $300{ }^{\circ} \mathrm{C}$ and above, depending on the growth rate. Our films become insulating at roughly the same temperatures, which suggests that our films also become stochiometric at these same temperatures. These trends at higher growth temperatures are qualitatively similar to the effects of extrinsic doping in the europium chalcogenides. ${ }^{5}$

Figure 3 shows the temperature dependence of the resistivity for three films, EuS67, EuS64, and EuS70, grown at 34,100 , and $200{ }^{\circ} \mathrm{C}$, respectively. These films were grown on untreated (100) GaAs substrates. Results from a film grown

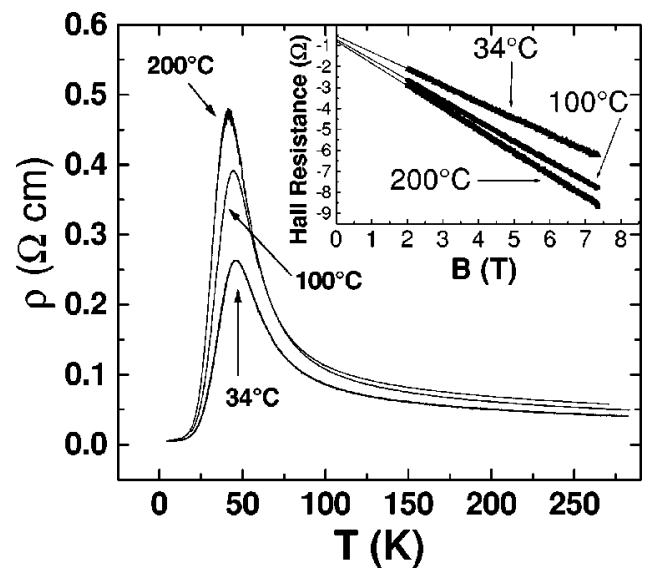

FIG. 3. $\rho$ vs $T$ data for films grown at the indicated substrate temperatures. The inset shows Hall data from 2 to $7.35 \mathrm{~T}$ with linear extrapolations to zero field for samples grown at the indicated substrate temperatures. The measuring temperature is $10 \mathrm{~K}$.

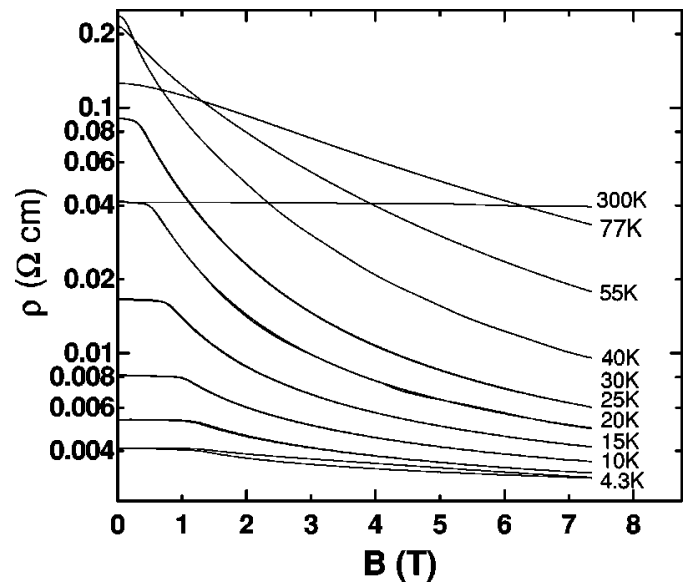

FIG. 4. MR curves for a sample grown at room temperature.

on a surface treated substrate will be shown later for comparison. The resistivity of these films also shows a similar dependence on the growth temperature as on extrinsic doping: the resistivity decreases with decreasing growth temperature while the temperature for the resistivity maximum, $T_{\rho \max }$, increases.

The magnetoresistance of these films shows behavior similar to extrinsically doped bulk crystals as well. As shown in Fig. 4, the MR which is negative in all of our measurements, peaks and reaches more than an order of magnitude at $T_{\rho \max }$. Around $T_{\rho \max }$ we observed a butterfly-type ${ }^{20}$ lowfield MR typical of a material with high spin polarization and a certain degree of granularity. At the lowest temperature of $4.3 \mathrm{~K}$, a hysteresis in the MR persists to our highest measuring field of $7.35 \mathrm{~T}$. We presently do not have an explanation for the origin of this high-field hysteresis, which renders the extraction of the Hall data at this temperature unreliable. Thus a direct comparison with earlier measurements on bulk EuS at liquid helium temperature are not possible. The inset in Fig. 3 shows the high field Hall data for the three samples at $T=10 \mathrm{~K}$. The carrier concentrations calculated from the high-field slope of the Hall data based on a one-band picture as a function of sample growth temperature are given in Table I. EuS has a band gap of $3.1 \mathrm{eV}$ (Ref. 21) with the $4 f$ electrons occupying a narrow, ${ }^{22}$ nonconducting band $1.7 \mathrm{eV}$ below the conduction band. Since the $4 f$ electrons would occupy any possible holes generated by surplus sulfur sites in the energetically more favorable valence band, it is therefore a reasonable assumption that the carriers in this material

TABLE I. Carrier concentrations for different growth temperatures.

\begin{tabular}{lcc}
\hline \hline Sample & $\begin{array}{c}\text { Carrier } \\
\text { concentration } \\
\left(\text { electrons } / \mathrm{cm}^{3}\right)\end{array}$ & $\begin{array}{c}\text { Substrate } \\
\text { growth } \\
\text { temperature }\end{array}$ \\
\hline EuS70 & $1.72 \times 10^{20}$ & $34^{\circ} \mathrm{C}$ \\
EuS64 & $1.37 \times 10^{20}$ & $100^{\circ} \mathrm{C}$ \\
EuS67 & $1.27 \times 10^{20}$ & $200^{\circ} \mathrm{C}$ \\
\hline \hline
\end{tabular}




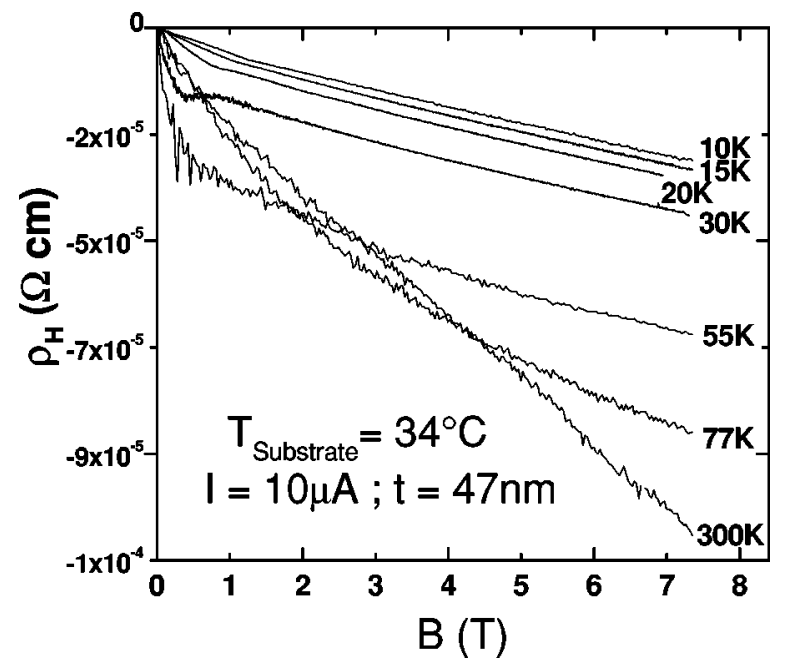

FIG. 5. Hall resistivity as a function of field for EuS70 at various temperatures.

consist solely of electrons. The measured Hall carrier concentration decreases with increasing growth temperature, which demonstrates that the variation in the film conductivity is due to changes in carrier concentration and possibly sulfur deficiencies of the films at lower growth temperatures. A sample grown at $300^{\circ} \mathrm{C}$ was also characterized but was too resistive to be measured reliably, suggesting its composition to be near that of stochiometric EuS.

All these magnetotransport data indicate that the effect of growth temperature on the film properties is similar to those of extrinsic doping. The growth temperature can clearly be used as an effective means of controlling the properties of the EuS films in spintronics device structures.

As shown in Fig. 3, linear extrapolation of the high-field Hall data for all three samples does not intersect the origin. This feature becomes more prominent at higher temperatures, as is depicted in Fig. 5, which shows a set of Hall data for EuS70 at various temperatures. Upon inspection of the curve, two features are apparent: the carrier concentration extracted from the high-field slope of the Hall data is independent of temperature even up to temperatures above $T_{\rho \max }$, and there is a change in slope at lower fields that becomes more dramatic with increasing film resistivity. Above $T_{\rho \max }$, the high-field slope changes noticeably. The carrier concentration as seen from the Hall data at 77 and $300 \mathrm{~K}$ (Fig. 5) appears to decrease from its lower temperature value at these higher temperatures. However, mean free path calculations show that the transport is no longer diffusive at temperatures near $T_{\rho \max }$ and above, and since $R_{0}$ does not obey the usual $R_{0}=(1 / n e)$ for hopping conduction, ${ }^{23,24}$ the carrier concentration from the data obtained in such a simplistic manner is not reliable. The values for $n$ at lower temperatures were calculated from the high-field Hall slope at fields in which transport is still diffusive. The presence of an AHE significantly above $T_{c}$ is surprising, however observation of an AHE above $T_{c}$ in the hopping regime has been observed previously in magnetic semiconductors. ${ }^{25}$

We now focus our attention on the low-field nonlinear component of the Hall data. Although the nonlinearity at low

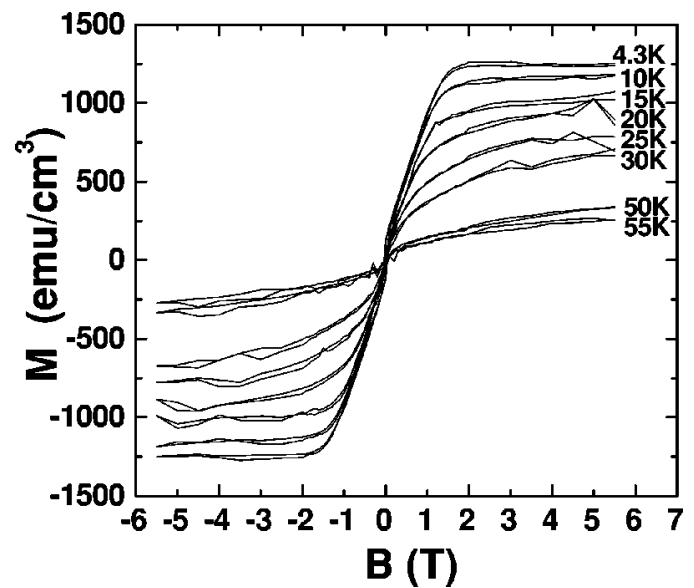

FIG. 6. Volume magnetization as a function of $B$ for EuS70. The noise at higher fields is due to measurement near zero moment.

fields could possibly reflect a change of carrier density with magnetic field, we will show in the following data that its correlation with the magnetic measurements and its scaling with $\rho$ suggest that the effect is an AHE.

The initial clue that the variation of the slope of the Hall data with field was related to the magnetization was due to its correlation with the demagnetization effect in the MR. The internal field $H_{i}$ in a thin film is given by $H_{i}=H_{A}$ $-N M$, where $H_{A}$ is the applied field and $N$ is the demagnetization factor $(N=1$ in the thin film limit). For thin films, $H_{A}$ is exactly compensated for by $M$ such that $H_{i}=0$ until the saturation magnetization $M_{S}$ is attained, while the magnetic induction $B=\mu_{0} H_{A}$. The effect is exhibited as a negligible MR until the saturation field is reached. We observed that at lower temperatures the change in slope of the Hall

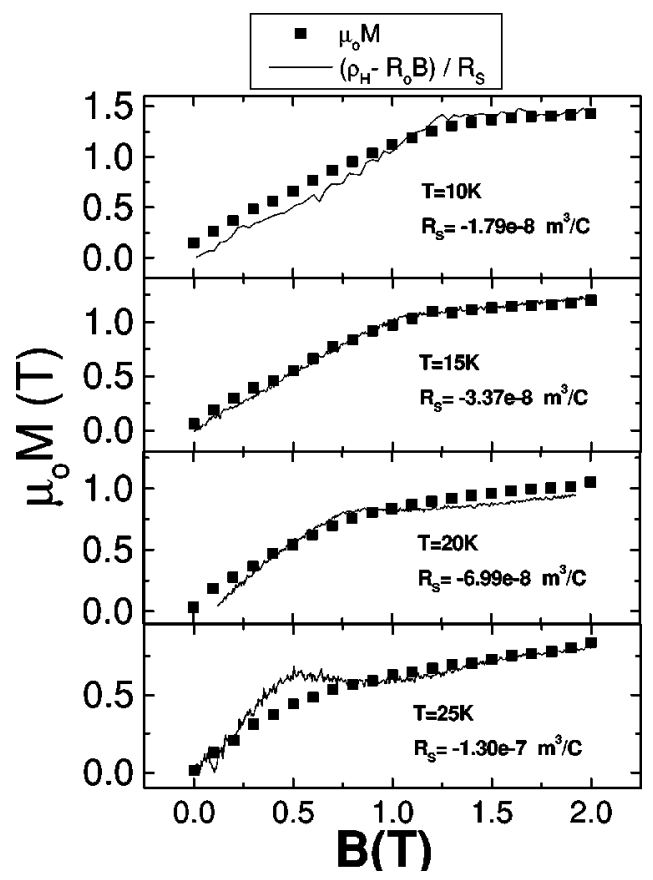

FIG. 7. $\left(\rho_{H}-R_{0} B\right)$ vs $B$ as plotted against $\mu_{0} M$ vs $B$ at various temperatures. 


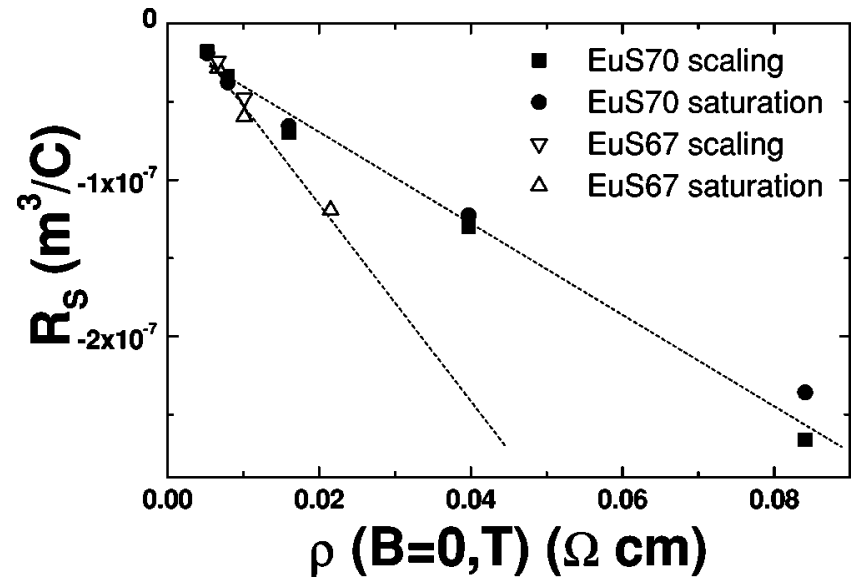

FIG. 8. Comparison of $R_{S}$ as extracted using different methods.

data with field occurred at the same field at which $\rho$ began to change. This field is approximately $1.6 \mathrm{~T}$, which is also the field we calculated to be the saturation field for stochiometric EuS.

A hallmark of the AHE is the linear scaling between the magnetization and the nonlinear component of the Hall data. In order to examine directly the correlation between the Hall data and the magnetization, we performed measurements of the film magnetization in a perpendicular field. The data for EuS70 are shown in Fig. 6. The diamagnetic background signal from the GaAs substrate has been subtracted. Since the carrier concentration derived from the high-field normal Hall effect is in excess of $10^{20} \mathrm{~cm}^{-3}$, considerably above degeneracy, no change in carrier number as a function of field is expected. Thus the linear component of the ordinary Hall effect may be subtracted using the high-field data. This results in a close linear scaling between the two sets of data, $\mu_{0} M(T)$ vs $\left(\rho_{H}-R_{0} B\right)$, at various temperatures as is demonstrated in Fig. 7. This is strong evidence that the nonlinear effect in the Hall data in these EuS thin films is an AHE. The AHE coefficient $R_{S}$ is the ratio of $\mu_{0} M$ and $\left(\rho_{H}-R_{0} B\right)$ as a function of $B$. It is also possible to extract $R_{S}$ by linearly extrapolating the high-field Hall data to zero field in order to obtain the maximum anomalous contribution to $\rho_{H}$. One then uses the saturation magnetization to calculate $R_{S}$. Due to the error in evaluating the saturation field from the magnetic and transport measurements, we believe the extrapolation method to be a less accurate procedure of obtaining $R_{S}$. A comparison of $R_{S}$ as a function of the zero applied field film resistivity $\rho(B=0, T)$ as extracted by these two procedures is shown in Fig. 8. Also shown are the data for a more resistive sample grown at $200{ }^{\circ} \mathrm{C}$, EuS67. In both cases the results are slightly different but are consistent: a linear relationship is evident between $R_{S}$ and $\rho(B=0, T)$. The linear relationship $(n=1)$ suggests that the AHE is due to skew scattering of the conduction electrons within the material.

The temperature dependence of the resistivity and the presence of a metal-insulator transition around $T_{c}$ have been explained by von Molnár and Kasuya ${ }^{7}$ as well as Shapira et $a l .{ }^{8}$ in EuTe with a picture based on formation of magnetic

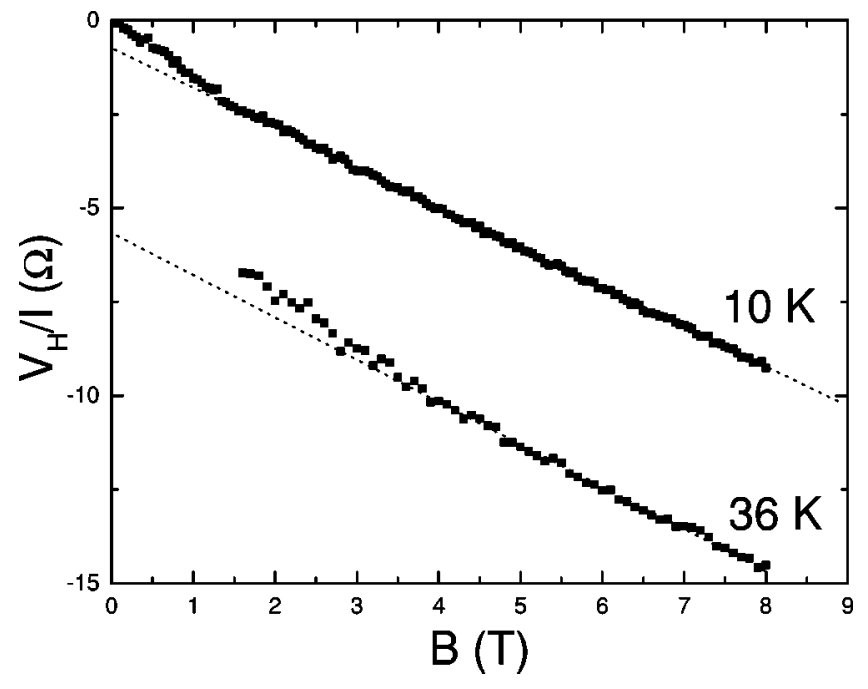

FIG. 9. Hall data from a more epitaxially grown film on (100) $\mathrm{Si}$ measured at the given temperatures. Data less than $1.5 \mathrm{~T}$ at $36 \mathrm{~K}$ could not be measured accurately enough to allow meaningful analysis.

polarons. The rapid decrease in resistivity below $T_{c}$ can be understood from overlapping of magnetic polarons and diminishing spin disorder scattering as $T$ decreases. A vanishing $R_{S}$ at low temperatures was also $\operatorname{seen}^{26}$ in $\mathrm{La}_{2 / 3} \mathrm{Sr}_{1 / 2} \mathrm{MnO}_{3}$, which suggests that the skew scattering and the vanishing $R_{S}$ originate from the same spin fluctuations. This could explain why the AHE is not significant in EuS single crystals at low temperatures, ${ }^{6}$ where even less compositional disorder is expected than in thin films. This scenario also implies that the AHE is intrinsic to EuS and not due to the structural disorder present in some of the thin films, a conjecture supported by our observation of AHE in the more epitaxial films grown on surface treated $\mathrm{Si}$ substrate. Figure 9 shows a set of Hall data from such a film: at temperatures below $10 \mathrm{~K}$ the nonlinear component in the Hall voltage is nearly absent but becomes very pronounced close to $T_{\rho \text { max }}$ $(35 \mathrm{~K})$.

\section{CONCLUSIONS}

We have carried out a systematic Hall measurement on a series of EuS thin films at different temperatures across the ferromagnetic transition. The carrier concentration inferred from the high-field Hall data correlates with the conductivities of the films grown at different substrate temperatures, which indicates that the variation of film conductivity with growth temperature is due to differences in carrier concentration, most likely resulting from varying degrees of sulfur deficiencies. We have observed an AHE in these films which has not been seen before. The anomalous Hall coefficient $R_{S}$ is found to scale linearly with $\rho$, which suggests the observed $\mathrm{AHE}$ is due to skew scattering.

\section{ACKNOWLEDGMENT}

This work was supported by the DARPA through ONR Grant Nos. N00014-99-1-1094 and MDA-972-02-1-0002. 
${ }^{1}$ S. A. Wolf, D. D. Awschalom, R. A. Buhrman, J. M. Daughton, S. von Molnár, M. L. Roukes, A. Y. Chtchelkanova, and D. M. Treger, Science 294, 1488 (2001).

${ }^{2}$ S. Datta and B. Das, Appl. Phys. Lett. 56, 665 (1990).

${ }^{3}$ G. Schmidt and L. W. Molenkamp, Physica E 10, 484 (2001).

${ }^{4}$ E. I. Rashba, Phys. Rev. B 62, R16 267 (2000).

${ }^{5}$ S. von Molnár and S. Methfessel, J. Appl. Phys. 38, 959 (1967).

${ }^{6}$ Y. Shapira and T. B. Reed, Phys. Rev. B 5, 4877 (1972).

${ }^{7}$ S. von Molnár and T. Kasuya, Phys. Rev. Lett. 21, 1757 (1968) (the observation of an AHE in this paper was incorrect).

${ }^{8}$ Y. Shapira, S. Foner, N. F. Oliveira, Jr., and T. B. Reed, Phys. Rev. B 5, 2647 (1972).

${ }^{9}$ L. Esaki, P. J. Stiles, and S. von Molnar, Phys. Rev. Lett. 19, 852 (1967).

${ }^{10}$ J. S. Moodera, X. Hao, G. A. Gibson, and R. Meservey, Phys. Rev. Lett. 61, 637 (1988).

${ }^{11}$ X. Hao, J. S. Moodera, and R. Meservey, Phys. Rev. B 42, 8235 (1990).

${ }^{12}$ P. LeClair, J. K. Ha, H. J. M. Swagten, J. T. Kohlhepp, C. H. van de Vin, and W. J. M. de Jonge, Appl. Phys. Lett. 80, 625 (2002).

${ }^{13}$ J. Kohne, G. Mair, N. Rasula, B. Saftic, and W. Zinn, J. Phys. (Paris), Colloq. 41, C5-127 (1980).

${ }^{14}$ S. Demokritov, U. Rucker, and P. Grunberg, J. Magn. Magn. Mater. 163, 21 (1996).
${ }^{15}$ K. Schienbein, G. Mair, and K. Reichelt, Thin Solid Films 52, L5 (1978).

${ }^{16}$ G. Busch, M. Campagna, and H. C. Siegmann, J. Appl. Phys. 42, 1781 (1971).

${ }^{17}$ S. Feierabend, U. Kischko, and R. Niedermayer, Phys. Status Solidi 34, K135 (1976).

${ }^{18}$ J. Keller, J. S. Parker, J. Stankiewicz, D. E. Read, P. A. Stampe, R. J. Kennedy, P. Xiong, and S. von Molnár, IEEE Trans. Magn. 38, 2673 (2002).

${ }^{19}$ For a review, see The Hall Effect and Its Applications, edited by C. L. Chien and C. R. Westgate (Plenum, New York, 1980), p. 44.

${ }^{20}$ J. O’Donnell, M. Onellion, M. S. Rzchowski, J. N. Eckstein, and I. Bozovic, Phys. Rev. B 55, 5873 (1997).

${ }^{21}$ D. E. Eastman, F. Holtzberg, and S. Methfessel, Phys. Rev. Lett. 23, 226 (1969).

${ }^{22}$ S. J. Cho, Phys. Rev. B 1, 4589 (1970).

${ }^{23}$ T. Holstein, Phys. Rev. 124, 1329 (1961).

${ }^{24}$ L. Friedman, Phys. Rev. 131, 2445 (1963).

${ }^{25}$ P. Matl, N. P. Ong, Y. F. Yan, Y. Q. Li, D. Studebaker, T. Baum, and G. Doubinina, Phys. Rev. B 57, 10248 (1998).

${ }^{26}$ J. C. Chen, S. C. Law, L. C. Tung, C. C. Chi, and W. Guan, Phys. Rev. B 60, 12143 (1999). 